\title{
La calidad percibida de servicio y su influencia en la fidelidad de usuarios mayores en centros de fitness públicos
}

\section{Influence of perceived service quality on loyalty among elderly users of public fitness centres}

\author{
Jerónimo García, Ainara Bernal, Alejandro Lara y Pablo Galán \\ Departamento de Educación Física y Deporte, Universidad de Sevilla, España
}

Disponible online 31 de agosto de 2013

\begin{abstract}
En la actualidad es patente el aumento de la población mayor en los servicios deportivos, lo cual hace necesario la evaluación de la percepción de calidad debido a su repercusión sobre la fidelidad. Por esta razón, los objetivos de este trabajo fueron conocer la percepción de calidad y la fidelidad en clientes mayores de 55 años en centros de fitness públicos. Analizar si existen diferencias significativas en la percepción de calidad de este grupo de población. Y, examinar si la calidad percibida incide en la fidelidad de estos clientes. Para ello se utilizó una muestra compuesta por 610 usuarios de centros de fitness públicos (406 mujeres y 204 hombres), realizándose un análisis factorial exploratorio, análisis de la fiabilidad, prueba t y ANOVA de un factor, y regresiones lineales. Los resultados muestran escalas fiables y válidas de calidad percibida de servicio (empleados, programas e instalaciones) y fidelidad, así como la existencia de diferencias significativas en las dimensiones de empleados e instalaciones en cuanto al género, edad y formación académica, y en la dimensión de programas en el género, formación académica, estado civil y duración de la sesión de práctica. Igualmente se constató la influencia positiva entre calidad percibida y fidelidad del cliente. Estos hallazgos sugieren la importancia de una buena gestión de la calidad.
\end{abstract}

Palabras clave: Calidad Percibida de Servicio; Fidelidad; Centros de Fitness; Mayores.

Nowadays, there is an increasing number of elderly people attending sports centres leading to interest in the evaluation of perceived quality due to its impact on loyalty. Thus, the objectives of this study/survey were to determine perceived quality and customer loyalty among users older than 55 years old attending public fitness centres, to analyze whether there were significant differences between users, and to examine if perceived quality influenced customer loyalty. We used a sample of 610 users of public fitness centres (406 women and 204 men), performing exploratory factor analysis, reliability analysis, t-test and one-way ANOVA, and linear regression. The results show reliable and valid scales of service quality (employees, programs, and facilities) and loyalty. Significant differences were found in the employees and facilities dimensions regarding gender, age, and educational background and in the programs dimension regarding gender, educational background, marital status, and duration of the practice session. A positive influence was found between quality and customer loyalty. These findings suggest the importance of good quality management.

Keywords: Service Quality; Loyalty; Fitness centres; elderly people.

Correspondencia: Jerónimo García Fernández, Departamento de Educación Física y Deporte, Universidad de Sevilla, C/Pirotecnia, s/n, 41013, Sevilla; E-mail: jeronimo@us.es. E-mail de los otros autores: Ainara Bernal: abernal1@us.es, Alejandro Lara: alelarboc@gmail.com, Pablo Galán: pablogalanlopez@gmail.com 
La consolidación de la industria del fitness como sector enmarcado dentro del ocio, ha provocado la demanda de profesionales con conocimientos en gestión deportiva y, en la actualidad, la calidad se ha convertido en una de las metas de las organizaciones (Chelladurai y Chang, 2000), llegando a constituirse departamentos responsables para la consecución de la misma. Esto es debido a que la calidad percibida de servicio se considera como un factor determinante para garantizar la buena salud empresarial que aumenta la capacidad competitiva y el crecimiento económico (Colmenares y Saavedra, 2007). Esta evolución fomenta que se lleven a cabo investigaciones cuyo objetivo sea identificar y determinar los aspectos importantes a tener en cuenta a la hora de la búsqueda del máximo rendimiento desde la calidad percibida de los servicios. En este sentido, los responsables de la calidad están interesados en conocer los elementos que son determinantes para conseguir una evaluación positiva del servicio por parte del usuario (Mañas, Jiménez, Mayor, Martínez-Tur y Moliner, 2008), ya que redundará en beneficios para la organización (Tschohl, 2001).

Igualmente, es importante resaltar el cambio cultural que se está produciendo en los hábitos deportivos de las personas mayores en España. Esto se ve reflejado en que en las últimas tres décadas se ha pasado de una casi nula práctica deportiva regular a una práctica de casi el 20\% (García Ferrando y Llopis, 2011). A pesar de que este porcentaje es el menor de todos los grupos de edad, es el que muestra una mayor regularidad en la práctica deportiva. Así pues, se prevé que continuarán produciéndose cambios cualitativos y cuantitativos en los hábitos deportivos de la población mayor a lo largo de los próximos años (García Ferrando y Llopis, 2011).

Por otro lado, la conceptualización y medición de la calidad percibida en el ámbito deportivo, también ha adquirido en los últimos años el mismo nivel de importancia y preocupación que en otros sectores económicos (Martínez, 2009) y para conocer con un poco más de profundidad la variable estudiada se realiza la revisión bibliográfica que se expone a continuación.

\section{La calidad percibida del servicio}

La definición de la calidad percibida ha sido el tema más debatido y con mayores controversias de la literatura del marketing (Brady y Cronin, 2001). El término "calidad percibida" ha tenido y tiene numerosas definiciones sin existir una definición única y global. Sin embargo, según Martínez y Martínez (2009) las definiciones de mayor aceptación y las más utilizadas son "juicio del consumidor sobre la excelencia o superioridad de un producto/servicio" (Zeithaml, 1988, p. 3), o "impresión global del consumidor sobre la relativa superioridad o inferioridad de una organización y sus servicios" (Bitner y Hubber, 1994, p.7).

Por su parte, Calabuig, Molina y Nuñez (2012, p. 68) la definen como "una evaluación a largo plazo post-consumo o post-uso, susceptible de cambio en cada transacción, fuente de desarrollo y cambio de las actitudes hacia el objeto de consumo o uso, y que es el resultado de procesos psicosociales de carácter cognitivo y afectivo". En este sentido, Nuviala y Casajús (2005, p. 4) nos recuerdan que "la calidad es diferente de cada persona, es un concepto muy dinámico, ya que está sometido a los cambios de necesidades, pareceres, preferencias y gustos de los usuarios".

En relación a la medición de la calidad percibida, la gran mayoría de estudios se han llevado a cabo utilizando la herramienta de medida SERVQUAL (Parasuraman, Zeithaml y Berry, 1988). A pesar de esto, manifiesta diferentes limitaciones, analizada también algunas de ellas, en el sector deportivo, del ocio y la recreación (Alexandris, Zahariadis, Tsorbatzoudis y Grouios, 2004; Calabuig, Quintanilla y Mundina, 2008; Kim y Kim, 1995; Kouthouris y Alexandris, 2005; Nuviala, Tamayo, Nuviala, González y Fernández, 2010). Nuviala, Tamayo, Iranzo y Falcón (2008) señalaban las limitaciones en el sector deportivo como un excesivo número de ítems, especificidad máxima que impide la generalización de resultados y la falta de dimensiones o factores indispensables en la valoración del servicio (Alexandris et al., 2004; Kim y Kim, 1995; Kouthouris et al., 2005). Del mismo modo, Calabuig et al. (2008) recogen que el instrumento de medida no es estable, ya que Taylor, Sharland, Cronin y Bullard (1993), demostraron que en los servicios de recreación, las cinco dimensiones del SERVQUAL son eventuales y poco específicas. Así pues, basándonos en las posibles limitaciones de las escalas existentes y por la falta de especificidad, en el ámbito de los servicios deportivos surgen diferentes instrumentos para la evaluación de la calidad percibida tal y como se puede observar en la Tabla 1. El estudio de la calidad, supone un importante paso en el sector deportivo pues su importancia radica en la conexión directa que tiene con la fidelidad del cliente (Baker y Crompton, 2000).

No obstante, la revisión del concepto fidelidad en la literatura no encuentra un consenso en la definición y en la medición del constructo. A pesar de esto, la definición más aceptada de fidelidad es la propuesta por Oliver (1999) que la entiende como "un profundo compromiso de recomprar un producto/ servicio preferido en el futuro, provocando así repetidas compras de la misma marca o conjunto de marcas, pese a que las influencias situacionales y los esfuerzos de marketing tengan el potencial de generar un cambio de comportamiento" (p. 34).

La teoría que fundamenta la calidad percibida del servicio como influyente en la fidelidad del cliente está refutada por numerosos autores (Calabuig, Burillo, Crespo, Mundina y Gallardo, 2010). El dilema aparece cuando se pretende determinar si esta relación es directa o indirecta a través de otros elementos como la satisfacción o el valor percibido. En este sentido, se encuentran autores que afirman la existencia de una repercusión directa de la calidad percibida sobre la leal- 
Tabla 1

Instrumentos de evaluación de la calidad percibida.

\begin{tabular}{|c|c|c|c|}
\hline Autores & Instrumento & Dimensiones & Aplicación \\
\hline Kim y Kim (1995) & QUESC & $\begin{array}{l}\text { 1. Ambiente } \\
\text { 2. Actitud del empleado } \\
\text { 3. Fiabilidad } \\
\text { 4. Información disponible } \\
\text { 5. El programa que ofrece } \\
\text { 6. Consideraciones personales } \\
\text { 7. El precio } \\
\text { 8. El privilegio } \\
\text { 9. La facilidad } \\
\text { 10. La estimulación } \\
\text { 11. La conveniencia }\end{array}$ & Centros deportivos \\
\hline $\begin{array}{l}\text { McDonald, Sutton y Milne } \\
\text { (1995) }\end{array}$ & $T E A M Q U A L$ & $\begin{array}{ll}\text { 1. } & \text { Tangibles } \\
\text { 2. Seguridad } \\
\text { 3. Empatía } \\
\text { 4. Fiabilidad } \\
\text { 5. Responsabilidad }\end{array}$ & $\begin{array}{l}\text { Equipos deportivos } \\
\text { profesionales }\end{array}$ \\
\hline $\begin{array}{c}\text { Howat, Crilley, Absher y } \\
\text { Milne (1996) }\end{array}$ & $C E R M-C S Q$ & $\begin{array}{l}\text { 1. Los servicios básicos } \\
\text { 2. La calidad del personal } \\
\text { 3. El servicio en general } \\
\text { 4. Los servicios secundarios }\end{array}$ & Servicios deportivos y de ocio \\
\hline $\begin{array}{l}\text { Luna-Arocas, Mundina y } \\
\text { Gómez (1998) }\end{array}$ & NEPTUNO- 1 & $\begin{array}{l}\text { 1. Las clases } \\
\text { 2. El tiempo libre } \\
\text { 3. La limpieza } \\
\text { 4. El entorno náutico } \\
\text { 5. La comida } \\
\text { 6. La comida complementaria } \\
\text { 7. El material náutico } \\
\text { 8. Los horarios } \\
\text { 9. La conserjería } \\
\text { 10. La teoría }\end{array}$ & Escuelas náuticas de la comunidad valenciana \\
\hline $\begin{array}{l}\text { Papadimitriou y Karteroliotis } \\
\qquad(2000)\end{array}$ & FITSSQ & $\begin{array}{l}\text { 1. Calidad del instructor, } \\
\text { 2. Atracción de la instalación y su funcionamiento } \\
\text { 3. La disponibilidad y entrega del programa } \\
\text { 4. Otros servicios }\end{array}$ & Fitness and Sport Service \\
\hline $\begin{array}{l}\text { Thedorakis, Kambitsis, Laios } \\
\text { y Koustelios (2001) }\end{array}$ & SPORTSERV & $\begin{array}{ll}\text { 1. } & \text { Acceso } \\
\text { 2. } & \text { Fiabilidad } \\
\text { 3. } & \text { Capacidad de respuesta } \\
\text { 4. Los tangibles } \\
\text { 5. La seguridad }\end{array}$ & Deportes profesionales \\
\hline $\begin{array}{l}\text { Morales, Hernández-Mendo y } \\
\text { Blanco, }(2005)\end{array}$ & $I C P A F$ & $\begin{array}{l}\text { 1. Profesor } \\
\text { 2. Respecto a las instalaciones } \\
\text { 3. Respecto a las actividades } \\
\text { 4. Respecto al personal e información }\end{array}$ & Programas de Actividad Física \\
\hline Dhurup, Singh y Surujlal, & HAFSQ & $\begin{array}{l}\text { 1. El personal } \\
\text { 2. La programación y los médicos } \\
\text { 3. La conveniencia y la difusión de la información } \\
\text { 4. La funcionalidad y el diseño } \\
\text { 5. El ambiente y la accesibilidad } \\
\text { 6. La atracción de la instalación } \\
\text { 7. La seguridad } \\
\text { 8. El apoyo y la adhesión }\end{array}$ & Centros deportivos \\
\hline $\begin{array}{l}\text { Rial, Varela, Rial y Real } \\
\text { (2010) }\end{array}$ & QSport-10 & $\begin{array}{l}\text { 1. Instalaciones } \\
\text { 2. Personal }\end{array}$ & Centros deportivos \\
\hline $\begin{array}{l}\text { Calabuig, Mundina y Crespo } \\
(2010)\end{array}$ & EVENTQUAL & $\begin{array}{l}\text { 1. Los tangibles } \\
\text { 2. El personal } \\
\text { 3. Los servicios complementarios } \\
\text { 4. La accesibilidad. }\end{array}$ & Eventos deportivos \\
\hline Gálvez y Morales (2011) & CECASDEP & $\begin{array}{l}\text { 1. Instalación deportiva } \\
\text { 2. Espacio deportivo } \\
\text { 3. Vestuario } \\
\text { 4. Programas de actividad física } \\
\text { 5. Profesor/a o monitor/a }\end{array}$ & Servicios municipales deportivos \\
\hline $\begin{array}{l}\text { García, Cepeda y Martín } \\
\text { (2012) }\end{array}$ & CALIDFIT & $\begin{array}{l}\text { 1. Personal } \\
\text { 2. Instalaciones } \\
\text { 3. Ambiente } \\
\text { 4. Número de equipamientos } \\
\text { 5. Servicios }\end{array}$ & Centros de fitness privados \\
\hline $\begin{array}{c}\text { Nuviala, Grao-Cruces, } \\
\text { Tamayo, Nuviala Álvarez y } \\
\text { Fernández-Martínez, en prensa }\end{array}$ & EPOD2 & $\begin{array}{l}\text { 1. Técnicos } \\
\text { 2. P. Servicios } \\
\text { 3. Comunicación } \\
\text { 4. Actividad } \\
\text { 5. Material } \\
\text { 6. Espacios }\end{array}$ & Calidad percibida en el sector deportivo \\
\hline
\end{tabular}


tad (Cronin, Brady y Hult, 2000; Cronin y Taylor, 1992). Así, Yacout (2010) encontró que la calidad percibida del servicio era el predictor más importante de la lealtad, seguido del valor percibido y por último, de los beneficios de la confianza. Por esta razón, y debido a la creciente importancia otorgada a la fidelidad del cliente en el sector deportivo, donde el usuario mayor es cada vez más practicante de actividad física (García Ferrando y Llopis, 2011), se hace necesaria la evaluación de la percepción de calidad para a posteriori, establecer pautas que mejoren la fidelidad en las instalaciones deportivas.

Así pues, este trabajo tiene los objetivos de: Conocer la percepción de calidad y la fidelidad en clientes mayores de 55 años en centros de fitness públicos. Analizar si existen diferencias significativas en la percepción de calidad de este grupo de población. Y, examinar si la calidad percibida incide en la fidelidad de estos clientes.

\section{Participantes \\ Método \\ Los datos corresponden a usuarios de centros de fitness públicos del municipio de Sevilla. Para la elección de los mismos, se optó por elegir a aquellos que ofertan como mínimo una sala de fitness con equipamiento de tonificación y cardio- vascular. Así, de los siete existentes, finalmente participaron seis. La muestra estuvo compuesta por 610 usuarios (406 muje- res y 204 hombres) con edades comprendidas entre los 55 y 80 años. De entre ellos, el $69.8 \%(n=426)$ tenía entre 55 y 70 años, y con más de 70 años el $30.2 \%(n=184)$.}

\section{Instrumentos}

La recogida de datos se llevó a cabo por un cuestionario como instrumento de medida. Para ello, en primer lugar se elaboró el cuestionario dividido en características del usuario (género, edad, formación académica, estado civil, permanencia en la instalación y duración de la sesión), una medida de 15 ítems para la calidad percibida de servicio elaborada a partir de la escala propuesta por Brady y Cronin (2001), y finalmente una medida para la fidelidad o intenciones de comportamiento de 4 ítems basada en Zeithaml, Berry y Parasuraman (1996). A continuación se reunió a dos expertos del área de organización de empresas, dos del área de educación física y deportes y tres gerentes de instalaciones deportivas para saber si los ítems estaban correctamente formulados y no presentaban dudas. Tras la revisión y no habiendo observado ningún problema, se solicitó de nuevo a un grupo de cinco clientes de estas organizaciones que se lo leyeran y dijeran si entendían y podrían contestarlo correctamente. Finalmente y al comprobar que no generaba dudas ni problemas de comprensión, se optó por utilizar dicho instrumento, cuya respuesta se medía a través de una escala tipo Likert con 9 opciones de respuesta $(1=$ totalmente en desacuerdo y $9=$ totalmente de acuerdo).

\section{Procedimiento}

La recogida de datos partió en primer lugar con una reunión con la dirección del Instituto Municipal de Deportes de Sevilla, solicitando el permiso para acceder a las instalaciones y poder administrar los cuestionarios. Posteriormente y después de la aprobación de la institución, se reunió a los dos grupos de encuestadores distribuyéndolos por instalaciones y horarios. En este sentido cada grupo tenía asignado tres instalaciones y debían recoger la información de los usuarios una vez finalizada la actividad deportiva en horario de mañana y de tarde, respondiendo a todas las preguntas que les pudieran realizar los participantes. La colaboración de los clientes fue voluntaria y anónima sin que hubiese ningún tipo de información que permitiese identificar a los usuarios, siendo la duración del cuestionario de unos 15 minutos.

Análisis de datos. Para llevar a cabo los objetivos del estudio, se procedió en primer lugar a un análisis de los descriptivos en relación a las características de la muestra. Seguidamente se realizó una validación de constructo de las medidas de calidad percibida de servicio y fidelidad del cliente con un análisis factorial exploratorio (AFE), siendo analizada la fiabilidad de las escalas a través del cálculo del alfa de Cronbach para el análisis de la consistencia interna. Para analizar si existían diferencias significativas entre las diferentes dimensiones halladas y las características de los participantes, se realizaron pruebas $t$ y ANOVA de un factor. Finalmente, para analizar la relación entre la calidad percibida de servicio y la fidelidad del cliente, se optó por el cálculo de regresiones lineales para establecer las relaciones entre las variables objeto de estudio, para de esta forma contrastar los datos referentes a los modelos que se establecieron al plantear los objetivos de este trabajo. Todos los análisis fueron realizados utilizando el programa estadístico SPSS 18.0.

\section{Resultados \\ Análisis descriptivo de los participantes}

Tras el análisis descriptivo (Tabla 2) se destaca cómo el $40.5 \%(n=247)$ tenía una formación elemental, y tan solo el $11.7 \%(n=71)$ formación universitaria, máster y/o doctorado. En relación al estado civil de los participantes, el $64.9 \%$ $(n=396)$ estaban casados frente al 6.4\% $(n=39)$ solteros. De igual modo, el 48.5\% $(n=296)$ afirmó que llevaba como usuario de los servicios deportivos menos de 2 años, siendo $26.7 \%$ ( $n=163)$ los que llevaban más de 4 años inscritos a los centros de fitness. Finalmente en relación a la duración de la sesión, se destaca el hecho de que el 88.5\% $(n=540)$ practicaran durante 1 hora frente al $0.5 \%(n=3)$ que lo hacen más de 2 horas. 
Tabla 2

Distribución de los participantes según formación académica, estado civil, permanencia en la instalación y duración de la sesión.

\begin{tabular}{|c|c|c|c|}
\hline & Variables & $n$ & Porcentaje (\%) \\
\hline \multirow{9}{*}{$\begin{array}{l}\text { Formación } \\
\text { académica }\end{array}$} & Elemental & 247 & 40.5 \\
\hline & Primaria & 179 & 29.3 \\
\hline & Secundaria & 60 & 9.8 \\
\hline & Universitaria & 67 & 11 \\
\hline & Formación profesional & 51 & 8.4 \\
\hline & Máster & 1 & 0.2 \\
\hline & Doctorado & 3 & 0.5 \\
\hline & Sin datos & 2 & 0.3 \\
\hline & Total & 610 & 100 \\
\hline \multirow{4}{*}{ Estado civil } & Casado & 396 & 64.9 \\
\hline & Soltero & 39 & 6.4 \\
\hline & Otro & 175 & 28.7 \\
\hline & Total & 610 & 100.0 \\
\hline \multirow{10}{*}{ Permanencia } & Menos de 6 meses & 78 & 12.8 \\
\hline & Entre 6 y 12 meses & 105 & 17.2 \\
\hline & Entre 13 y 18 meses & 24 & 3.9 \\
\hline & Entre 19 y 24 meses & 89 & 14.6 \\
\hline & Entre 25 y 30 meses & 15 & 2.5 \\
\hline & Entre 31 y 36 meses & 102 & 16.7 \\
\hline & Entre 37 y 42 meses & 3 & 0.5 \\
\hline & Entre 43 y 48 meses & 31 & 5.1 \\
\hline & Más de 48 meses & 163 & 26.7 \\
\hline & Total & 610 & 100.0 \\
\hline \multirow{6}{*}{$\begin{array}{l}\text { Duración de la } \\
\text { sesión }\end{array}$} & Menos de 1 hora & 8 & 1.3 \\
\hline & 1 hora & 540 & 88.5 \\
\hline & 1,5 horas & 10 & 1.6 \\
\hline & 2 horas & 49 & 8.0 \\
\hline & Más de 2 horas & 3 & 0.5 \\
\hline & Total & 610 & 100.0 \\
\hline
\end{tabular}

\section{Análisis de la estructura interna de las escalas}

En la Tabla 3 quedan recogidos todos los datos que a continuación se comentan. Se realizó un AFE sobre los 15 ítems de la escala de calidad percibida por el método de extracción de componentes principales y posterior rotación Varimax. Previamente, se calculó la medida de adecuación muestral de KaiserMeyer-Olkin (KMO) y el test de esfericidad de Barlett, mostrando en el primer caso un valor de $.880 \mathrm{y}$ el segundo estadísticamente significativo $\left(\mathrm{X}_{105}=8327.336 ; p<.001\right)$, por lo que dicho análisis era pertinente. El AFE resultó en 3 dimensiones (instalaciones, personal y programa) que explicaron el $76.53 \%$ del total de la varianza. La dimensión instalación se compuso de los ítems del 1 al 5, la dimensión personal del 6 al 10, y programas del 11 al 15. En relación a la escala de fidelidad, el AFE resultó en un solo factor que explicó el $87.05 \%$ de la varianza total, obteniendo un valor de .841 para Kaiser-Meyer-Olkin y significativo en el test de esfericidad de Barlett $\left(X_{6}=2583.276\right.$; $p<.001)$.

En relación al análisis de la fiabilidad, la escala de calidad percibida de servicio obtuvo una alfa de Cronbach de .905. En ningún caso se tuvo que eliminar ningún ítem para mejorar dicho valor. Referente a la escala de fidelidad del cliente, los resultados también mostraron una alta fiabilidad $(\alpha=.949)$ no teniendo que eliminar ningún ítem para incrementarla.
Tabla 3

Estructura factorial rotada, fiabilidad y valores de KMO en la Escala de Calidad Percibida de Servicio y Fidelidad del Cliente.

\begin{tabular}{|c|c|c|c|c|}
\hline \multicolumn{5}{|c|}{ Subescala de Escala de Calidad percibida de servicio } \\
\hline Items & 1 factor & 2 factor & 3 factor & $\alpha \sin$ ítem \\
\hline $\begin{array}{l}\text { Las instalaciones del centro } \\
\text { deportivo son atractivas }\end{array}$ & & & .807 & .903 \\
\hline $\begin{array}{l}\text { Las instalaciones del centro } \\
\text { deportivo son espaciosas. }\end{array}$ & & & .884 & .900 \\
\hline $\begin{array}{l}\text { Las instalaciones del centro } \\
\text { deportivo están limpias. }\end{array}$ & & & .831 & .900 \\
\hline $\begin{array}{l}\text { El equipamiento del centro } \\
\text { deportivo está en buenas } \\
\text { condiciones. }\end{array}$ & & & .874 & .900 \\
\hline $\begin{array}{l}\text { El ambiente del centro deportivo } \\
\text { (temperatura, iluminación, ruido y } \\
\text { olor) es bueno. }\end{array}$ & & & .809 & .902 \\
\hline $\begin{array}{l}\text { Los empleados responden } \\
\text { rápidamente a las necesidades de } \\
\text { los clientes. }\end{array}$ & \multicolumn{2}{|l|}{.843} & & .897 \\
\hline $\begin{array}{l}\text { Los empleados trabajan con } \\
\text { entusiasmo. }\end{array}$ & \multicolumn{2}{|l|}{.818} & & .896 \\
\hline Los empleados son educados. & \multicolumn{2}{|l|}{.847} & & .896 \\
\hline $\begin{array}{l}\text { Los empleados ayudan a que los } \\
\text { clientes se sientan cómodos. }\end{array}$ & \multicolumn{2}{|l|}{.801} & & .896 \\
\hline Los empleados son expertos. & \multicolumn{2}{|l|}{.755} & & .900 \\
\hline $\begin{array}{l}\text { Los programas de actividad física } \\
\text { me ayudan a incrementar mi } \\
\text { energía. }\end{array}$ & \multicolumn{2}{|r|}{.725} & & .897 \\
\hline $\begin{array}{l}\text { Los programas de actividad física } \\
\text { me ayudan a mejorar mi salud. }\end{array}$ & \multicolumn{2}{|r|}{.857} & & .902 \\
\hline $\begin{array}{l}\text { Los programas de actividad física } \\
\text { me ayudan a mejorar mi humor }\end{array}$ & \multicolumn{2}{|r|}{.739} & & .900 \\
\hline $\begin{array}{l}\text { Los programas de actividad física } \\
\text { me ayudan a mejorar mi bienestar } \\
\text { psicológico. }\end{array}$ & \multicolumn{2}{|r|}{.829} & & .902 \\
\hline $\begin{array}{l}\text { Los programas de actividad física } \\
\text { me ayudan a mejorar mi nivel de } \\
\text { condición física. }\end{array}$ & \multicolumn{2}{|r|}{.825} & & .898 \\
\hline Varianza explicada & \multirow[t]{2}{*}{48.52} & \multirow[t]{2}{*}{20.220} & 7.78 & 76.530 \\
\hline KMO (Kaiser-Meyer-Olkin) & & & & .880 \\
\hline Alfa de Cronbach & .930 & .910 & .906 & .905 \\
\hline \multicolumn{5}{|c|}{ Subescala Fidelidad del cliente } \\
\hline & \multicolumn{2}{|c|}{1 factor } & \multicolumn{2}{|c|}{$\alpha$ sin ítem } \\
\hline $\begin{array}{l}\text { Realizaré comentarios positivos a } \\
\text { un amigo acerca de los programas y } \\
\text { servicios de este centro deportivo. }\end{array}$ & \multicolumn{2}{|c|}{.950} & \multicolumn{2}{|c|}{.924} \\
\hline $\begin{array}{l}\text { Si me preguntan, recomendaré este } \\
\text { centro deportivo. }\end{array}$ & \multicolumn{2}{|c|}{.955} & \multicolumn{2}{|c|}{.922} \\
\hline $\begin{array}{l}\text { Continuaré participando en los } \\
\text { programas de este centro deportivo. }\end{array}$ & \multicolumn{2}{|c|}{.919} & \multicolumn{2}{|c|}{.940} \\
\hline $\begin{array}{l}\text { Me volvería a inscribir en este } \\
\text { centro deportivo si me diera de baja. }\end{array}$ & \multicolumn{2}{|c|}{.907} & \multicolumn{2}{|c|}{.946} \\
\hline Varianza explicada & & & \multicolumn{2}{|c|}{87.05} \\
\hline KMO (Kaiser-Meyer-Olkin) & & & \multicolumn{2}{|c|}{.841} \\
\hline Alfa de Cronbach & & & \multicolumn{2}{|c|}{.949} \\
\hline
\end{tabular}

\section{Análisis de la calidad percibida y fidelidad según caracterís-} ticas de los participantes

En primer lugar se procedió a resumir la escala de fidelidad y cada dimensión de la calidad percibida en una sola variable aunando los ítems correspondientes a cada factor (programas: $M=8.66, D T=.98$; empleados: $M=8.57, D T=1.11$; instalación: $M=7.39, D T=1.50$; fidelidad: $M=8.64, D T=1.08$ ). Posteriormente tal y como se puede observar en la tabla 4, se comprobó como existían diferencias significativas en relación al género y las dimensiones empleados, programas, instalacio- 
nes y fidelidad $(p<.001)$, siendo en todos los casos más positivas las percepciones en las mujeres $(M=8.61 ; M=8.67 ; M=$ $7.41 ; M=8.66)$. En relación a la edad, los resultados mostraron diferencias significativas en las dimensiones empleados e instalaciones $(p<.05)$, situándose las percepciones más positivas en los clientes de 71 a 75 años $(M=8.79 ; M=7.68)$ y las peores en los de 55 a 65 años $(M=8.29 ; M=6.96)$. Igualmente, se encontraron diferencias significativas referidas a la formación académica y las dimensiones de empleados y programas $(p<$ $.001)$ y de instalaciones $(p<.01)$. En este caso, las puntuaciones más elevadas se situaron en aquellos que tenían primaria en los empleados y los programas $(M=8.72 ; M=8.85)$ y universitaria en las instalaciones $(M=7.78)$. Finalmente, se encontraron diferencias significativas en la dimensión programas con el estado civil y en la duración de la sesión $(p<.05)$, existiendo mayores puntuaciones en los casados $(M=8.73)$ y los que practicaban menos de 1 hora $(M=8.70)$.

\section{Análisis de la relación entre la calidad percibida de servicio y la fidelidad del cliente}

Para examinar la relación existente entre la calidad percibida de servicio, sus dimensiones y la fidelidad del cliente, se realizó un análisis de regresión lineal. Los resultados mostraron que la calidad percibida tenía una correlación positiva con la fidelidad del cliente $\left(r^{2}=.358 ; p<.001\right)$. De igual modo, la dimensión que obtuvo una mayor correlación con la fidelidad fue "programas" $\left(r^{2}=.297 ; p<.001\right)$, seguido de "empleados" $\left(r^{2}=.296 ; p<.001\right)$ y con menor peso las instalaciones $\left(r^{2}=\right.$ $.082 ; p<.001)$.

\section{Discusión}

Este trabajo tenía los objetivos de conocer la percepción de la calidad percibida y la fidelidad de clientes en centros de fitness públicos, analizar si existían diferencias significativas entre los usuarios y examinar si la calidad percibida

Tabla 4

Diferencias en calidad percibida y fidelidad del cliente en función del género, edad, formación académica, estado civil, permanencia y duración de la sesión.

\begin{tabular}{|c|c|c|c|c|c|c|c|c|c|}
\hline \multirow[t]{2}{*}{ Variables } & & \multicolumn{2}{|c|}{ Empleados } & \multicolumn{2}{|c|}{ Programas } & \multicolumn{2}{|c|}{ Instalaciones } & \multicolumn{2}{|c|}{ Fidelidad } \\
\hline & & $M(D T)$ & $F(t)$ & $M(D T)$ & $F(t)$ & $M(D T)$ & $F(t)$ & $M(D T)$ & $F(t)$ \\
\hline \multirow[t]{2}{*}{ Género } & Hombre & $8.49(1.15)$ & $(4.61 * * *)$ & $8.63(.98)$ & $(2.18 * * *)$ & $7.33(1.59)$ & $(3.34 * * *)$ & $8.62(.85)$ & $(1.19 * * *)$ \\
\hline & Mujer & $8.61(1.08)$ & & $8.67(.98)$ & & $7.41(1.61)$ & & $8.66(1.18)$ & \\
\hline \multirow[t]{5}{*}{ Edad } & De 55 a 65 años & $8.29(1.42)$ & $3.21 *$ & $8.60(.93)$ & .088 & $6.96(1.70)$ & $3.17^{*}$ & $8.48(1.44)$ & 1.29 \\
\hline & De 61 a 65 años & $8.66(.98)$ & & $8.73(.82)$ & & $7.30(1.69)$ & & $8.61(1.13)$ & \\
\hline & De 66 a 70 años & $8.50(1.27)$ & & $8.50(1.37)$ & & $7.48(1.66)$ & & $8.65(.99)$ & \\
\hline & De 71 a 75 años & $8.79(.69)$ & & $8.82(.55)$ & & $7.68(1.35)$ & & $8.74(1.05)$ & \\
\hline & Más de 75 años & $8.66(.81)$ & & $8.70(.67)$ & & $7.52(1.34)$ & & $8.80(.47)$ & \\
\hline \multirow{7}{*}{$\begin{array}{l}\text { Formación } \\
\text { académica }\end{array}$} & Elemental & $8.58(1.12)$ & $4.73 * * *$ & $8.63(.96)$ & $6.59 * * *$ & $7.19(1.54)$ & $2.87 * *$ & $8.63(1.17)$ & .96 \\
\hline & Primaria & $8.72(.94)$ & & $8.85(.67)$ & & $7.50(1.79)$ & & $8.76(.92)$ & \\
\hline & Secundaria & $8.45(1.21)$ & & $8.65(.82)$ & & $7.53(1.60)$ & & $8.57(1.30)$ & \\
\hline & Universitaria & $8.48(1.11)$ & & $8.67(.64)$ & & $7.78(1.13)$ & & $8.61(.88)$ & \\
\hline & Formación profesional & $8.51(1.20)$ & & $8.37(1.46)$ & & $7.37(1.50)$ & & $8.50(1.13)$ & \\
\hline & Máster & $9.00(.00)$ & & $.00(.00)$ & & $6.80(.00)$ & & $6.75(.00)$ & \\
\hline & Sin datos & $4.60(1.13)$ & & $6.60(3.39)$ & & $3.70(1.27)$ & & $9.00(.00)$ & \\
\hline \multirow[t]{3}{*}{ Estado civil } & Casado & $8.62(1.10)$ & 1.31 & $8.73(.85)$ & $3.21 *$ & $7.35(1.65)$ & .31 & $8.65(1.12)$ & .39 \\
\hline & Soltero & $8.62(.69)$ & & $8.41(1.51)$ & & $7.46(1.30)$ & & $8.50(1.32)$ & \\
\hline & Otro & $8.46(1.18)$ & & $8.55(1.08)$ & & $7.45(1.56)$ & & $8.66(.91)$ & \\
\hline \multirow[t]{10}{*}{ Permanencia } & Menos de 6 meses & $8.51(1.36)$ & .44 & $8.64(.84)$ & .80 & $7.65(1.58)$ & 1.34 & $8.56(1.07)$ & .35 \\
\hline & Entre 6 y 12 meses & $8.59(1.06)$ & & $8.64(1.00)$ & & $7.51(1.55)$ & & $8.73(.81)$ & \\
\hline & Entre 13 y 18 meses & $8.28(1.26)$ & & $8.55(.88)$ & & $6.86(1.89)$ & & $8.69(.54)$ & \\
\hline & Entre 19 y 24 meses & $8.56(.98)$ & & $8.52(1.24)$ & & $7.28(1.58)$ & & $8.66(.83)$ & \\
\hline & Entre 25 y 30 meses & $8.40(1.35)$ & & $8.37(1.83)$ & & $7.22(1.75)$ & & $8.66(.81)$ & \\
\hline & Entre 31 y 36 meses & $8.65(.85)$ & & $8.82(.54)$ & & $7.20(1.67)$ & & $8.60(1.34)$ & \\
\hline & Entre 37 y 42 meses & $9.00(.00)$ & & $9.00(.00)$ & & $8.00(.40)$ & & $9.00(.00)$ & \\
\hline & Entre 43 y 48 meses & $8.53(1.00)$ & & $8.64(.80)$ & & $6.98(1.49)$ & & $8.83(.44)$ & \\
\hline & Más de 48 meses & $8.61(1.20)$ & & $8.68(1.03)$ & & $7.51(1.57)$ & & $8.60(1.33)$ & \\
\hline & Total & $8.57(1.11)$ & & $8.66(.98)$ & & $7.39(1.60)$ & & $8.64(1.08)$ & \\
\hline \multirow{4}{*}{$\begin{array}{l}\text { Duración de la } \\
\text { sesión }\end{array}$} & 1.5 horas & $8.72(.88)$ & & $7.82(2.82)$ & & $7.12(1.18)$ & & $8.27(.98)$ & \\
\hline & 2 horas & $8.34(1.16)$ & & $8.49(.85)$ & & $7.24(1.53)$ & & $8.61(.74)$ & \\
\hline & Más de 2 horas & $8.00(1.73)$ & & $8.13(1.33)$ & & $7.26(1.80)$ & & $7.66(2.30)$ & \\
\hline & Total & $8.57(1.11)$ & & $8.66(.98)$ & & $7.39(1.60)$ & & $8.64(1.08)$ & \\
\hline
\end{tabular}


incidía en la fidelidad de estos clientes. En este sentido, los resultados presentados aportan un conocimiento decisorio en la gestión de este tipo de instalaciones, planteando las bases para la comprensión de estos constructos en la industria del fitness.

Para comenzar con el primer hallazgo, el perfil de este tipo de instalaciones se decanta mayormente por mujeres al igual que afirman Pinillos (2004), Sanz, Redondo, Gutiérrez y Cuadrado (2005) y Bodet (2006), situándose el mayor porcentaje en edades comprendidas entre los 61 y 70 años. Esta situación nos hace pensar que cada vez más los usuarios mayores están más concienciados con la práctica deportiva y que a su vez, las mujeres comienzan a incorporarse más decisoriamente a estas actividades tal y como afirman García Ferrando y Llopis (2011). Asimismo, el perfil del usuario mayor en centros de fitness públicos se decanta por un cliente con formación elemental al contrario de lo que ocurre en centros de fitness privados (García, Sañudo, Martín y Cepeda, 2011), casados, que practica de media una hora por sesión y su permanencia en la instalación se sitúa en los dos años. En este sentido, en el trabajo de García et al. (2011) se puso de manifiesto que la población mayor en centros de fitness privados tenía una permanencia media superior a los cuatro años, lo que hace pensar que en instalaciones públicas no se estén teniendo en cuenta programas de fidelización de clientes, o que no existan barreras de salida de las instalaciones al contrario como ocurre en las privadas. No obstante, es destacable que sí aparezcan estas diferencias que redundan directamente en que las organizaciones públicas deban hacer un mayor esfuerzo en captar clientes mayores.

Por otro lado en relación a la calidad percibida, en la mayoría de los casos los trabajos que se han realizado se han basado en analizar las dimensiones que forman el constructo y su relación con la satisfacción (Calabuig et al., 2010). Así, a diferencia de lo propuesto por García et al. (2012) sobre la multidimensionalidad en seis dimensiones, nuestros resultados apoyan la formación del constructo por tres dimensiones denominadas empleados, programas e instalaciones, al igual que afirmaron Brady y Cronin (2001). De igual modo, la escala ofreció altos valores de validez y fiabilidad por lo que se postula como una herramienta de fácil aplicación en el contexto de los centros de fitness públicos. Concretamente, de entre las tres dimensiones resultantes, empleados fue la que mayor varianza explicó del concepto calidad percibida, seguida de programas y en última instancia, instalaciones. Así, estos hallazgos corroboran la tradición en la investigación sobre calidad percibida, poniendo su prioridad en la interacción de los empleados con los clientes (Parasuraman, Berry y Zeithmal, 1991). Por esta razón, nuestros resultados sugieren que los directores han de prestar una mayor atención en primer lugar a los empleados, y una menor prioridad a la instalación al contrario que para Mañas et al. (2008), donde el equipamiento y la aparatología que disponía la instalación obtenían una mayor importancia frente a los programas y a los recursos humanos de la organización deportiva.
De igual modo, la escala de fidelidad del cliente resultó en una sola dimensión mostrando una fiabilidad y validez elevada. Asimismo, al igual que ocurre con la escala de calidad percibida presentada, la medida de fidelidad se ofrece como un instrumento sencillo de utilizar por los directores, por lo que se refuerza su aplicabilidad práctica.

Este trabajo también destaca las diferencias que pueden existir entre los usuarios en cuanto a su percepción y a las características de los mismos. Justamente, nuestros resultados apuestan porque las mujeres de 71 a 75 años con una formación primaria, son las que valoran más positivamente a los empleados frente a los hombres de 55 a 65 años que disponen de un doctorado. Igualmente, los programas son valorados más positivamente por las mujeres casadas con formación primaria que practican durante 1 hora, al contrario de lo que ocurre con los hombres solteros con doctorado que practican durante 1.5 horas. Referido a las instalaciones, las mujeres de 71 a 75 años con formación universitaria fueron las que percibieron más positivamente los elementos que conforman el centro de fitness frente a los hombres de 55 a 65 años con formación elemental. En este sentido, estos hallazgos muestran la prioridad para los gerentes de estas instalaciones en conocer más detenidamente por qué perciben estos perfiles mejor y peor las dimensiones que conforman la calidad percibida.

Así, detectar dichos factores se postula como necesario ya que esta situación redundará en una mayor fidelidad de sus clientes. Es por ello que, siguiendo a Pedragosa y Correia (2009) sobre la prioridad en instalaciones deportivas por investigar la lealtad de sus usuarios, nuestro trabajo recoge unos hallazgos realmente importantes sobre la fidelidad del cliente. Precisamente nuestros resultados apoyan la relación entre la calidad percibida y la fidelidad del cliente, en donde los programas y los empleados fueron los más decisorios para que un cliente permaneciera fiel. En este sentido, los estudios de Oh, Lee y Kim (2011) también recogían como la fidelidad de los usuarios de centros fitness venía determinada por los empleados donde sus capacidades técnicas y habilidades interpersonales eran la fuente de diferenciación de servicios, satisfacción y en última instancia, la retención de clientes.

Sin embargo, tal y como se recoge en cualquier trabajo de investigación, nuestro estudio no está exento de limitaciones. En este sentido, el número más limitado de dimensiones en la calidad percibida frente a otras medidas propuestas, resta detectar bajas puntuaciones en otras dimensiones y por lo tanto ser más minucioso en el conocimiento del cliente. Asimismo, aunque son escasos los estudios que ponen de manifiesto la percepción exclusiva de clientes mayores en centros de fitness, se convierte en una restricción ya que se cierra a la percepción de una tipología de usuario, al igual que ocurre por haberse realizado en instalaciones públicas y en un momento concreto. No obstante, si bien estas limitaciones ayudan a comprender la complejidad de un campo en su comienzo de investigación, abren nuevas puertas de investigaciones con las que se pretende 
conocer mejor a un consumidor cada vez más exigente. Es por ello que se ofrecen como futuras líneas, el estudio de diferentes perfiles de clientes en centros de fitness privados y su relación con la fidelidad. De igual modo, es interesante comparar si la percepción de perfiles de organizaciones públicas y privadas difiere, analizándolos a su vez en diferentes momentos temporales.

\section{Referencias}

1. Alexandris, K., Zahariadis, P., Tsorbatzoudis, C. y Grouios, G. (2004). An empirical investigation of the relationships among service quality, customer satisfaction and psychological commitment in a health club context. European Sport Management Quarterly, 4, 36-52. http://dx.doi. org/10.1080/16184740408737466

2. Baker, D. A. y Crompton, J. L. (2000). Quality, satisfaction and behavioral intentions. Annals of Tourism Research, 27, 785-804. http://dx.doi.org/10.1016/S0160-7383(99)00108$\underline{5}$

3. Bitner, M. J. y Hubbert, A. R. (1994). Encounter satisfaction versus overall satisfaction versus quality: The consumer's voice. En R. T. Rust y R. L. Oliver. (Eds.), Service Quality: New Directions in Theory and Practice (pp. 72-94). London: Sage Publications.

4. Brady, M. K. y Cronin, J. J. (2001). Some new thoughts on conceptualizing perceived service quality: a hierarchical approach. Journal of Marketing, 65, 34-49. http://dx.doi. org/10.1509/jmkg.65.3.34.18334

5. Bodet, G. (2006). Investigating customer satisfaction in a health club context by an application of the tetraclasse model. European Sport Management Quarterly, 6, 149165. http://dx.doi.org/10.1080/16184740600954148

6. Calabuig, F., Burillo, P., Crespo, J., Mundina, J.J. y Gallardo, L. (2010). Satisfacción, calidad y valor percibido en espectadores de atletismo. Revista Internacional de Medicina y Ciencias de la Actividad Física y el Deporte, 10, 577-593.

7. Calabuig, F., Molina, N., y Núñez, J. (2012). Una aplicación inicial del modelo tridimensional de calidad de servicio en centros deportivos privados. E-balonmano. Journal Sport of Science, 8, 67-81.

8. Calabuig, F., Mundina, J. y Crespo, J. (2010). Eventqual: una medida de la calidad percibida por los espectadores de eventos deportivos. Retos. Nuevas tendencias en Educación Física, Deporte y Recreación, 18, 66-70.

9. Calabuig, F., Quintanilla, I. y Mundina, J. J. (2008). La calidad percibida de los servicios deportivos: Diferencias según instalación, género, edad y tipo de usuario en servicios náuticos. Revista Internacional de Ciencias del Deporte, 4, 25-43. http://dx.doi.org/10.5232/ricyde2008.01003

10. Chelladurai, P. y Chang, K. (2000). Targets and Standards of Quality in Sport Services. Sport Management Review, 3, 1-22. http://dx.doi.org/10.1016/S1441-3523(00)70077-5
11. Colmenares, O. A. y Saavedra, J. L. (2007). Aproximación teórica de los modelos conceptuales de la calidad del servicio. Revista Técnica Administrativa. Buenos Aires, 6, 138175.

12. Cronin, J. J., Brady, M. K. y Hult, G. T. (2000). Assessing the effects of quality, value and customer satisfaction on consumer behavioral intentions in service environments. Journal of Retailing, 76, 193-218. http://dx.doi. org/10.1016/S0022-4359(00)00028-2

13. Cronin, J. J. y Taylor, S. A. (1992). Measuring Service Quality: A Reexamination and Extension. Journal of Marketing, 56, 55-68. http://dx.doi.org/10.2307/1252296

14. Dhurup, M., Singh, P. C. y Surujlal, J. (2006). Customer service quality at commercial health and fitness centres. South African journal for research in sport, physical education and recreation, 28, 39-54.

15. Gálvez, P. y Morales Sánchez, V. (2011). Evaluación de la calidad en programas municipales deportivos: generalizabilidad y optimización de diseños de medida. Cuadernos de Psicología del Deporte, 11, 123-130.

16. García Ferrando, M. y Llopis, M. (2011). Encuesta sobre los hábitos deportivos en España. Ideal democrático y bienestar personal. Madrid: CSD.

17. García, J., Cepeda, G. y Martín, D. (2012). La satisfacción de clientes y su relación con la percepción de calidad en Centro de Fitness: utilización de la escala CALIDFIT. Revista de Psicología del Deporte, 21, 309-319.

18. García, J., Sañudo, B., Martín, D. y Cepeda, G. (2011). Perfil del usuario mayor en centros de fitness privados. Trabajo presentado en el $4^{\circ}$ Congreso Internacional de Actividad Físico Deportiva para Mayores, Marzo, Málaga.

19. Howat, G., Crilley, G., Absher, J. y Milne, L. (1996). Measuring customer service quality in sport and leisure centers. Managing Leisure, 1, 77-89. http://dx.doi. org/10.1080/136067196376456

20. Kim, D. y Kim, S. Y. (1995). QUESC: An instrument for assessing the service quality of sport centers in Korea. Journal of Sport Management, 9, 208-220.

21. Kouthouris, C. y Alexandris, K. (2005). Can service quality predict customer satisfaction and behavioral intentions in the sport tourism industry? An application of the SERVQUAL model in an outdoors setting. Journal of Sport \& Tourism, 10, 101-111. http://dx.doi. org/10.1080/14775080500223165

22. Luna-Arocas, R., Mundina, J. y Gómez, A. (1998). La creación de una escala para medir la calidad de servicio y la satisfacción: el Neptuno-1. En J. Martínez del Castillo (Ed.), Deporte y Calidad de Vida (pp. 279-290). Madrid: Librerías deportivas Esteban Sanz.

23. Mañas, M. A., Jiménez, G., Mayor, J. M., Martínez-Tur, V. y Moliner, C. P. (2008). Los tangibles como predictores de la satisfacción del usuario en servicios deportivos. Psicothema, 20, 243-248. 
24. Martínez, J. A. (2009). Estudio de la invarianza de escala mediante el método de cálculo integral en la medición de la calidad percibida de los servicios deportivos. RYCIDE, Revista Internacional de Ciencias del Deporte, 15, 17-35.

25. Martínez, J. A. y Martínez, L. (2009). La calidad percibida en servicios deportivos; mapas conceptuales de marca. Revista Internacional de Medicina y Ciencias de la Actividad Física y el Deporte, 9, 232-253.

26. McDonald, M. A., Sutton, W. A. y Milne, G. R. (1995). TEAMQUAL TM: Measuring service quality in professional team sports. Sport Marketing Quarterly, 4, 9-15.

27. Morales, V., Hernández-Mendo, A. y Blanco, A. (2005). Evaluación de la calidad de los programas de Actividad Física. Psicothema, 17, 311-317.

28. Nuviala, A. y Casajús, J. A. (2005). Calidad percibida del servicio deportivo en edad escolar desde la perspectiva de los padres. El caso de la provincia de Huelva. Revista Internacional de Medicina y Ciencias de la Actividad Física y el Deporte, 5, 1-12.

29. Nuviala, A., Grao-Cruces, A., Tamayo, J. A., Nuviala, R., Álvarez, J. y Fernández-Martínez, A. (en prensa). Diseño y análisis del cuestionario de valoración de servicios deportivos (EPOD2). Revista Internacional de Medicina y Ciencias de la Actividad Física y del Deporte.

30. Nuviala, A., Tamayo, J. A., Iranzo, J. y Falcón, D. (2008). Creación, diseño, validación y puesta en práctica de un instrumento de medición de la satisfacción de usuarios de organizaciones que prestan servicios deportivos. Retos, Nuevas tendencias en Educación Física, Deporte y Recreación, 14, 10-16.

31. Nuviala, A., Tamayo, J. A., Nuviala, R., González, J. A. y Fernández, A. (2010). Propiedades psicométricas de la escala de valoración de organizaciones deportivas EPOD. Retos. Nuevas tendencias en Educación Física, Deporte y Recreación, 18, 82-87.

32. Oh, S. H., Lee, H. S. y Kim, S. J. (2011). The Effects of Service Quality and Relationship Benefits on Relationship Commitment and Customer Loyalty in Membership Fitness Club. Journal of Global Scholars of Marketing Science, 21, 115-125. http://dx.doi.org/10.1080/21639159.2011.97110 $\underline{18}$

33. Oliver, R. L. (1999). Whence consumer loyalty? Journal of Marketing, 63, 33-44. http://dx.doi.org/10.2307/1252099

34. Papadimitriou, D. y Karteroliotis, K. (2000). The service quality expectations in private sport and fitness centers: a reexamination of the factor structure. Sport Marketing Quarterly, 9, 157-164.

35. Parasuraman, A., Zeithaml, V. y Berry, L. (1988). SERVQUAL: A Multi Item Scale for Measuring Consumer Perception of Service Quality. Journal of Retailing, 64, 12-40.
36. Parasuraman, A., Berry, L. L. y Zeithaml, V. A. (1991). Refinement and reassessment of the SERVQUAL scale. Journal of Retailing, 67, 420-450.

37. Pedragosa, V. y Correia, A. (2009). Expectations, satisfaction and loyalty in health and fitness clubs. International Journal of Sport Management and Marketing, 5, 450 - 464. http://dx.doi.org/10.1504/IJSMM.2009.023371

38. Pinillos, J. (2004). Winning the retention battle: Librando la batalla por la retención. Investigación y Marketing, 83, $39-42$.

39. Rial, J., Varela, J., Rial, A. y Ral, E. (2010). Modelización y medida de la Calidad Percibida en centros deportivos: la escala QSport-10. Revista Internacional de Ciencias del Deporte, 18, 57-73. http://dx.doi.org/10.5232/ ricyde2010.01804

40. Sanz, I., Redondo, J. C., Gutiérrez, P. y Cuadrado, G. (2005). La satisfacción en los practicantes de spinning: elaboración de una escala para su medición. Motricidad, European Journal of Human Movement, 13, 17-36.

41. Taylor, S. A., Sharland, A., Cronin, J. J. y Bullard, W. (1993). Recreational service quality in the international setting. Journal of Service Industry Management, 4, 68-86. http://dx.doi.org/10.1108/09564239310044316

42. Theodorakis, N., Kambitis, C., Laios, A. y Koustelios A. (2001). Relationship between measures of service quality and satisfaction of spectators in professional sports. Managing Service Quality, 11, 431-438. http://dx.doi. org/10.1108/09604520110410638

43. Tschohl, J. (2001). Servicio al cliente. El arma secreta de la empresa que alcanza la excelencia. México: Editorial Pax México.

44. Yacout, O. (2010). Service Quality, Relational Benefits, and Customer Loyalty in a Non-Western Context. Society for the Advancement of Management, 75, 4-22.

45. Zeithaml, V. A. (1988). Consumer perceptions of price, quality, and value: a means-end model and synthesis of evidence. Journal of Marketing, 52, 2-22. http://dx.doi. org/10.2307/1251446

46. Zeithaml, V. A., Berry, L. y Parasuraman, A. (1996). The Behavioral Consequences of Service Quality. Journal of Marketing, 60, 31-46. http://dx.doi.org/10.2307/1251929

Fecha de recepción: 20 de febrero de 2013

Fecha de recepción de la versión modificada: 13 de mayo de 2013 Fecha de aceptación: 18 de junio de 2013 